As for the isomorphism, we need only consider the case where $V$ is defined from $G$. Obviously, the correspondence $a b^{-1} \rightleftarrows a b$ is one-one from $G$ onto $V$. Moreover, the correspondent of $a b+c d$ is $a h^{-1}$ where $d c^{-1}=h b^{-1}$ or $h^{-1}=b^{-1} c d^{-1}$. This establishes the isomorphism.

HARVARD UNIVERSITY

\title{
AN INVARIANT OF INTERSECTION OF TWO SURFACES
}

\section{CHUAN-CHIH HSIUNG}

1. Introduction. Projective invariants of several pairs of surfaces have been deduced and characterized geometrically by various authors. ${ }^{1}$ In this paper we shall supplement their investigations by studying in ordinary space two surfaces intersecting at an ordinary point with distinct tangent planes.

In $\$ 2$ we show by analysis the existence of a projective invariant determined by the neighborhood of the second order of the two surfaces at the point of intersection.

The final two sections are devoted to the presentation of projectively, as well as metrically, geometric characterizations of this invariant.

2. Derivation. Suppose that two surfaces $S_{1}, S_{2}$ in ordinary space intersect at an ordinary point $O$ with distinct tangent planes $\tau_{1}, \tau_{2}$, and let the common tangent $t$ be distinct from the asymptotic tangents. Let $t_{1}, t_{2}$ be the harmonic conjugate lines of $t$ with respect to the asymptotic tangents of the surfaces $S_{1}, S_{2}$, respectively, at the point $O$. If we choose the point $O$ to be the origin, the lines $t, t_{2}, t_{1}$ to be, respectively, the axes $x, y, z$ of a general nonhomogeneous projective coordinate system, then the power series expansions of the surfaces $S_{1}, S_{2}$ in the neighborhood of the point $O$ may be written in the form

$$
\begin{aligned}
& S_{1}: \quad y=l_{1} x^{2}+m_{1} z^{2}+\cdots, \\
& S_{2}: \quad z=l_{2} x^{2}+m_{2} y^{2}+\cdots .
\end{aligned}
$$

Presented to the Society, September 13, 1943; received by the editors June 15, 1943.

${ }^{1}$ See the bibliography at the end of the paper. 
Let us now consider the most general projective transformation of coordinates which leaves the lines $t, t_{1}, t_{2}$ unchanged:

$$
\left\{\begin{array}{l}
x=a_{22} x^{*} /\left(1+a_{12} x^{*}+a_{13} y^{*}+a_{14} z^{*}\right), \\
y=a_{33} y^{*} /\left(1+a_{12} x^{*}+a_{13} y^{*}+a_{14} z^{*}\right), \\
z=a_{44} z^{*} /\left(1+a_{12} x^{*}+a_{13} y^{*}+a_{14} z^{*}\right),
\end{array}\right.
$$

where $a_{i k}$ are arbitrary constants. The effect of this transformation on equations (1), (2) is to produce two other equations of the same form whose coefficients, indicated by stars, are given by the formulas

$$
\begin{cases}a_{33} l_{1}^{*}=\underset{a_{22}^{2} l_{1},}{2} & a_{33} m_{1}^{*}=\underset{a_{44}}{2} m_{1}, \\ a_{44} l_{2}^{*}=a_{22}^{2} l_{2}, & a_{44} m_{2}^{*}=a_{33}^{2} m_{2},\end{cases}
$$

from which it follows immediately that

$$
I=l_{1}^{3} m_{2} / l_{2}^{3} m_{1}
$$

is an invariant determined by the neighborhoods of the second order of the two surfaces $S_{1}, S_{2}$ at the point $O$.

3. A projectively geometric characterization. Let us consider two pairs of planes, one of which is determined by the line $t_{2}$ and the asymptotic tangents of the surface $S_{1}$ at the point $O$, and the other by the line $t_{1}$ and the asymptotic tangents of the surface $S_{2}$ at the point $O$. The lines of intersection of these four planes lie on two planes passing through the line $t$ :

$$
\left(l_{1} m_{2}\right)^{1 / 2} y \pm\left(l_{2} m_{1}\right)^{1 / 2} z=0 .
$$

On the other hand, we observe that the plane sections of the surfaces $S_{1}, S_{2}$ made by any plane through the line $t$ have, in general, an ordinary contact at the point $O$. In particular, we can uniquely determine a plane through the line $t$ such that the plane sections have contact of the third order at the point $O$. The equation of this plane is easily found to be

$$
l_{2} y-l_{1} z=0 .
$$

From equations (6), (7) it follows at once that the double ratio of the four planes $\tau_{1}, \tau_{2},(7),(6)$ is equal to

$$
\left(0, \infty,-l_{1} / l_{2}, \pm\left(l_{2} m_{1} / l_{1} m_{2}\right)^{1 / 2}\right)= \pm I^{1 / 2} .
$$

Hence we obtain the following characterization of the invariant $I$ :

Suppose that two surfaces $S_{1}, S_{2}$ in ordinary space intersect at an ordinary point $O$ with distinct tangent planes $\tau_{1}, \tau_{2}$, and let the common 
tangent $t$ be distinct from the asymptotic tangents. Let $t_{1}, t_{2}$ be the harmonic conjugate lines of $t$ with respect to the asymptotic tangents of the surfaces $S_{1}, S_{2}$, respectively, at the point $O$; and let $\pi_{1}$ be one of the two planes through $t$ on which lie the lines of intersection of two pairs of planes, one of which is determined by $t_{2}$ and the asymptotic tangents of the surface $S_{1}$ at the point $O$, and the other by $t_{1}$ and the asymptotic tangents of the surface $S_{2}$ at the point $O$. If $\pi_{2}$ be the unique plane through the line $t$ such that the plane sections of the surfaces $S_{1}, S_{2}$ made by $\pi_{2}$ have contact of the third order at the point $O$, then the projective invariant $I$ associated with the surfaces $S_{1}, S_{2}$ at the point $O$ is equal to the square of the double ratio $\left(\tau_{1} \tau_{2}, \pi_{2} \pi_{1}\right)$.

4. A metrically geometric characterization. It is deemed worthwhile to find a simple metrical characterization of the projective invariant $I$. For this purpose let us make a projective transformation which leaves the point $O$ unchanged and carries the lines $t, t_{1}, t_{2}$ into three mutually perpendicular lines $\bar{t}, \bar{t}_{1}, \bar{t}_{2}$. Let $\bar{S}_{1}, \bar{S}_{2}$ be the transformed surfaces of $S_{1}, S_{2}$, and $\bar{x}, \bar{y}, \bar{z}$ the nonhomogeneous Cartesian coordinates of a point in space referred to the orthogonal coordinate system determined by the lines $\bar{t}, \bar{t}_{1}, \bar{t}_{2}$; then the power series expansions of the surfaces $\bar{S}_{1}, \bar{S}_{2}$ in the neighborhood of the point $O$ may be written in the form

$$
\begin{array}{ll}
\bar{S}_{1}: & \bar{y}=\bar{l}_{1} \bar{x}^{2}+\bar{m}_{1} \bar{z}^{2}+\cdots, \\
\bar{S}_{2}: & \bar{z}=\bar{l}_{2} \bar{x}^{2}+\bar{m}_{2} \bar{y}^{2}+\cdots,
\end{array}
$$

and the projective invariant $I$ takes the form

$$
I=\bar{l}_{1}^{3} \bar{m}_{2} / \bar{l}_{2}^{3} \bar{m}_{1} \text {. }
$$

Let $\bar{K}_{1}, \bar{K}_{2}$ be the total curvatures of the surfaces $\bar{S}_{1}, \bar{S}_{2}$ at the point $O$; and $\bar{R}_{1}, \bar{R}_{2}$ the radii of curvature at the point $O$ of the curves in which the tangent planes $\bar{z}=0, \bar{y}=0$ intersect with the surfaces $\bar{S}_{1}, \bar{S}_{2}$ respectively. Then it can be demonstrated that

$$
\begin{cases}\bar{K}_{1}=4 \bar{l}_{1} \bar{m}_{1}, & \bar{K}_{2}=4 \bar{l}_{2} \bar{m}_{2}, \\ \bar{R}_{1}=1 / 2 \bar{l}_{1}, & \bar{R}_{2}=1 / 2 \bar{l}_{2},\end{cases}
$$

and therefore that

$$
I=\bar{R}_{2}^{4} \bar{K}_{2} / \bar{R}_{1}^{4} \bar{K}_{1}
$$

Thus we obtain the following metrical characterization of the projective invariant $I$.

Let $K_{1}, K_{2}$ be the total curvatures of the surfaces $S_{1}, S_{2}$ at the point $O$, 
and $R_{1}, R_{2}$ the radii of curvature at $O$ of the curves in which the tangent planes $\tau_{2}, \tau_{1}$ intersect the surfaces $S_{1}, S_{2}$, respectively. Then $R_{2}^{4} K_{2} / R_{1}^{4} K_{1}$ is the projective invariant $I$ of the surfaces $S_{1}, S_{2}$ above indicated.

\section{BIBLIOGRAPHY}

1. E. Bompiani, Invarianti proiettivi di una particolare coppia di elementisuperficiali del $2^{\circ}$ ordine, Bollettino della Unione Matematica Italiana vol. 14 (1935) pp. 237-243.

2. P. Buzano, Invariante proiettivo di una particolare coppia di elementi di superficie, Bollettino della Unione Matematica Italiana vol. 14 (1935) pp. 93-98.

3. C. C. Hsiung, Projective invariants of a pair of surfaces, to appear in Amer. J. Math.

4. M. Mascalchi, Un nuovo invariante proiettivo di contatto di due superficie, Bollettino della Unione Matematica Italiana vol. 13 (1934) pp. 45-49.

5. R. Mehmke, Einige Sätze iiber die räumliche Collineation und Affinität, welche sich auf die Krïmmung von Kurven und Flächen beziehen, Schlömilchs Zeitschrift für Mathematik und Physik vol. 36 (1891) pp. 56-60.

6. R. Mehmke, Über zwei die Krümmung von Kurven und das Gauss'sche Krilmmungsmass von Flächen betreffende charakteristische Eigenschaften der linearen Punkttransformationen, ibid. vol. 36 (1891) pp. 206-213.

National University of Chekiang 